OLIVEIRA, A.P.G. et al. Uso de enriquecimentos ambientais como mitigadores de comportamentos anormais: uma revisão. PUBVET, Londrina, V. 8, N. 7, Ed. 256, Art. 1697, Abril, 2014.

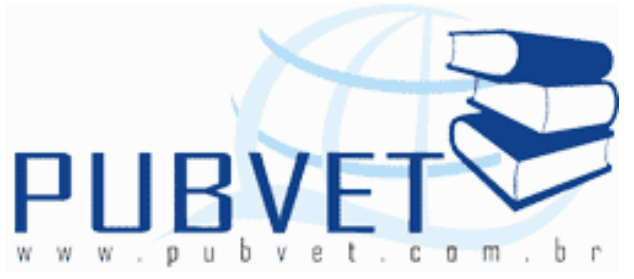

PUBVET, Publicações em Medicina Veterinária e Zootecnia.

\title{
Uso de enriquecimentos ambientais como mitigadores de comportamentos anormais: uma revisão
}

\begin{abstract}
Ana Paula Guedes Oliveira ${ }^{1}$, Weliton Menário Costa ${ }^{1}$, Rafael Nunes de Almeida ${ }^{1}$, Willian Moreira da Costa ${ }^{1}$, Natália Caroliny da Silva Dias ${ }^{1}$, Bárbara de Cássia Ribeiro Vieira ${ }^{1}$, Aparecida de Fátima Madella-Oliveira ${ }^{2}$, Célia Raquel Quirino ${ }^{3}$

${ }^{1}$ Alunos do curso de Licenciatura em Ciências Biológicas do Instituto Federal de Educação, Ciência e Tecnologia do Espírito Santo - Campus de Alegre, ES. ${ }^{2}$ Professora do Instituto Federal do Espírito Santo - Campus de Alegre, ES.

3 Professora da Universidade Estadual do Norte Fluminense, Campos dos Goytacazes, RJ.
\end{abstract}

\section{Resumo}

Esta revisão apresenta conceitos referentes à problematização do ambiente de cativeiro. O objetivo foi analisar a forma com a qual a utilização de enriquecimentos ambientais tem contribuído no desempenho natural de animais cativos. Conclui-se que essa técnica reduz o estresse, aumentando o grau de bem-estar e, consequentemente, estimulando o desenvolvimento de comportamentos típicos da espécie.

Palavras-chave: Bem-estar, comportamento, enriquecimento ambiental. 
OLIVEIRA, A.P.G. et al. Uso de enriquecimentos ambientais como mitigadores de comportamentos anormais: uma revisão. PUBVET, Londrina, V. 8, N. 7, Ed. 256, Art. 1697, Abril, 2014.

\title{
Use of environmental enrichments as mitigators of abnormal behavior: a review
}

\begin{abstract}
This review presents concepts concerning the problematization of captive environment. The objective was to analyze the way in which the use of environmental enrichment has contributed to the natural performance of captive animals. It is concluded that this technique reduces stress, increasing the degree of welfare and thus stimulating the development of behaviors typical of the species.
\end{abstract}

Keywords: Welfare, behavior, environmental enrichment.

\section{Introdução}

O ser humano provavelmente domesticou os primeiros animais de produção há aproximadamente dez mil anos, no Oriente Médio e na Ásia (MADELLA-OLIVEIRA et. al., 2011), dando início a um marcante processo de alteração entre a interação dos produtores e seus animais ao longo da história (MOLENTO, 2005).

Com o passar do tempo, o sistema de criação de animais passou a ser direcionado para um confinamento intensivo, com altas densidades populacionais. Este tipo inadequado de manejo, bem como suas implicações negativas para o bem-estar animal, são questões que foram levantados por Fraser e Broom (2002).

Por ser o ambiente de cativeiro diferente daquele no qual a espécie evoluiu (SGAI, 2007) e sendo, este, carente em atrativos que estimulem os aspectos físicos e mentais desses indivíduos, o bem-estar passa a ser afetado, ou seja, reduzido, acarretando consequências negativas, como o desenvolvimento de comportamentos anormais típicos de animais estressados (MALAFAIA et. al., 2011). 
OLIVEIRA, A.P.G. et al. Uso de enriquecimentos ambientais como mitigadores de comportamentos anormais: uma revisão. PUBVET, Londrina, V. 8, N. 7, Ed. 256, Art. 1697, Abril, 2014.

Para reduzir os estímulos que causam essas respostas estressoras e incentivar a execução de comportamentos típicos ou naturais do individuo, de forma não estereotipada, existe uma prática que vem sendo utilizada em alguns ambientes de cativeiro denominada de enriquecimento ambiental (MENDONÇA-FURTADO, 2006).

Diante dessas questões que abordam a utilização dos enriquecimentos como ferramenta utilizada para garantir o bem-estar animal e a corrente redução na execução de comportamentos anormais, justifica-se a realização desta revisão, que objetivou analisar a forma com a qual a utilização de enriquecimentos ambientais tem contribuído no desempenho natural de animais cativos.

\section{Bem-estar}

A ciência do bem-estar animal emerge como um mecanismo que leva o ser humano a rever as práticas até então existentes na produção animal, pois considera primordial o compromisso com o respeito e a ética do individuo (RAMOS, 2006).

A área de bem-estar animal vem despertando, ao longo do tempo, grande interesse em pesquisadores e instituições, apresentando um acentuado crescimento nos últimos anos em função da preocupação com a relação existente entre os organismos e a interação destes com o meio ambiente, visando à melhoria na qualidade de vida do animal (PIZZUTTO; SGAI; GUIMARÃES, 2009; CAMPOS, 2010).

Contudo, ainda existem inúmeras discussões com intuito de definir e quantificar o bem-estar, pois este deve ter relações com vários outros conceitos, tais como necessidades, felicidade, liberdade, adaptação, controle, capacidade de previsão, sentimentos, dor, ansiedade, medo, sofrimento, tédio, estresse e saúde (BROOM; MOLENTO, 2004; PIZZUTTO; SGAI; GUIMARÃES, 2009). 
OLIVEIRA, A.P.G. et al. Uso de enriquecimentos ambientais como mitigadores de comportamentos anormais: uma revisão. PUBVET, Londrina, V. 8, N. 7, Ed. 256, Art. 1697, Abril, 2014.

Segundo Broom (1986), o bem-estar de um indivíduo é a forma como ele se encontra ao tentar adaptar-se ao meio ambiente. De forma mais esclarecida, pode-se considerar que esse conceito refere-se ao estado como o animal reage ao passar por determinada fase de sua vida, ou situação inesperada (BROOM, 2001).

A definição de bem-estar aceita internacionalmente foi estabelecida em 1993 por FAWC (Farm Animal Welfare Council), na Inglaterra, que aborda o fato de os animais possuírem cinco liberdades (MOURA et. al., 2010) descritas conforme Mench (1998) (Tabela 1).

Dentre os comportamentos naturais mais realizados pelos animais, destacam-se os de comer e beber, por esta razão a primeira das cinco liberdades é a premissa "livre de fome e sede" (KYRIAZAKIS; TOLKAMP, 2011).

Tabela 1. Cinco liberdades pertencentes aos animais.

\section{LIBERDADES}

1. Liberdade nutricional: acesso à água fresca e uma dieta que satisfaça a saúde e vigor;

2. Liberdade sanitária: prevenção e ou diagnóstico de dor, doenças e feridas;

3. Liberdade comportamental: precisam ser livres para expressar comportamentos normais, ou seja, típicos da sua espécie;

4. Liberdade psicológica: ausência de ansiedade e medo;

5. Liberdade ambiental: ambiente apropriado com abrigo e área para descanso.

Fonte: Adaptada de Kyriazakis; Tolkamp (2011).

A Organização Mundial da Saúde Animal (OIE), através do Código Sanitário para Animais Terrestres (2012), no artigo 7.1.2 estabelece dois princípios que garantem as cinco liberdades e o consequente bem-estar animal: 1. Existe uma relação crítica entre a saúde e o bem-estar animal; 2. As cinco liberdades são as orintações para alcançar o bem-estar. 
OLIVEIRA, A.P.G. et al. Uso de enriquecimentos ambientais como mitigadores de comportamentos anormais: uma revisão. PUBVET, Londrina, V. 8, N. 7, Ed. 256, Art. 1697, Abril, 2014.

Nos casos em que as necessidades básicas, como alimento, água, abrigo e cuidados não são respeitadas, o grau de bem-estar dos animais fica comprometido de forma significativa, podendo levá-los ao adoecimento e até mesmo à morte (MUNRO; MUNRO, 2008; KYRIAZAKIS; TOLKAMP, 2011).

Todas essas concepções referentes ao bem-estar devem ser levadas em consideração para que ocorra o máximo possível de interações benéficas entre os indivíduos e o meio. No entanto, diversos problemas são elencados em função de os indivíduos estarem submetidos a uma rotina diferente daquela na qual seria natural à espécie, sendo preciso mensurar o grau de bem-estar que pode variar de alto a baixo grau, a fim de propor soluções que minimizem o sofrimento e melhorem a qualidade de vida dos animais (MARANHÃO et. al., 2006; MACHADO et. al., 2007; BURN et. al., 2010; HAMMERSCHMIDT, 2012).

Métodos diagnósticos de bem-estar devem considerar quatro questões fundamentais: praticidade, confiabilidade, repetitibilidade e fatores externos que podem influenciar na mensuração do quadro animal (BUTTERWORTH et. al., 2011).

Por ser incluído em uma escala que varia de muito alto a muito baixo (BROOM; FRASER, 2010), o grau de bem-estar deve ser medido de forma objetiva para detectar as informações que levarão a tomadas de medidas éticas diante de determinadas situações (BROOM; MOLENTO, 2004). Essas medidas são descritas na Tabela 2, conforme os relatos de Broom e Johnson (2000).

É extremamente importante relatar que o baixo grau de bem-estar no animal aumenta as chances do aparecimento de doenças (COCKRAM; HUGUES, 2011).

O Ministério da Agricultura, Pecuária e Abastecimento (MAPA) publicou no diário oficial a Instrução Normativa $n^{\circ} 56$, de 2008, que direciona as orientações de boas práticas de bem-estar para animais de produção, destacando o cuidado com o manejo animal, para reduzir o estresse e evitar sofrimentos (BRASIL, 2008). 
OLIVEIRA, A.P.G. et al. Uso de enriquecimentos ambientais como mitigadores de comportamentos anormais: uma revisão. PUBVET, Londrina, V. 8, N. 7, Ed. 256, Art. 1697, Abril, 2014.

Tabela 2. Medidas de grau de bem-estar.

MEDIDAS DE ELEVADO GRAU DE BEM-ESTAR
MEDIDAS DE REDUZIDO GRAU DE BEM-ESTAR

\begin{tabular}{llll}
\hline & & Expectativa de vida, bem como \\
crescimento e reprodução reduzidos;
\end{tabular}

Fonte: Adaptada de Broom e Johnson (2000).

Com base nestas ideias, adotou-se o estresse, que pode ser bom ou ruim, como critério para medir o bem-estar do individuo. O estresse é considerado bom quando o animal consegue manter um equilíbrio entre ele e o meio, ao reagir diante de determinadas situações, podendo, então, ser levado ao processo chamado de adaptação (MACHADO FILHO; HOTZEL, 2000).

Por outro lado, o estresse é considerado ruim quando for crônico, pois nesta fase, o animal aprende que reagir a certas situações não ocasiona adaptação, então desiste de reagir, entrando em um processo chamado desistência aprendida e como consequência, ocorre a redução da eficácia do sistema imunológico, a diminuição da produtividade (MACHADO FILHO; HOTZEL, 2009) e da capacidade cognitiva, levando ao aparecimento de comportamentos anormais (MENDL et. al., 2001). 
OLIVEIRA, A.P.G. et al. Uso de enriquecimentos ambientais como mitigadores de comportamentos anormais: uma revisão. PUBVET, Londrina, V. 8, N. 7, Ed. 256, Art. 1697, Abril, 2014.

\section{Comportamento anormal}

\subsection{Definição}

Através da prática de criação intensiva de animais de produção, onde alguns permaneciam isolados e/ou em instalações fechadas e com espaço reduzido, ocorreu uma alteração drástica em seus comportamentos naturais, ocasionando situações de estresse (SILVA et al., 2008), que acarretaram em comportamentos atípicos, também definidos como anômalos ou anormais.

Comportamento anormal são ações compensatórias em respostas a ambientes empobrecidos e pouco estimulantes que, por não permitirem a expressividade de comportamentos normais ou específicos da espécie, estimulam o aparecimento de outros diferentes daqueles que os animais demonstrariam em vida livre, como agressividade, automutilação, hipersexualidade, movimentos estereotipados, apatia, roer ou lamber partes da baia, desenvolver quadros depressivos, que podem até mesmo levar a morte (MASON, 1991; MAESTRIPIERI et. al., 1992; ALMEIDA, 2008; MALAFAIA et. al., 2011).

Estereotipias são movimentos irrelevantes, sem nenhum objetivo aparente, que se repetem de forma regular e atuam como atitudes compensatórias em resposta as frustrações provenientes de um ambiente empobrecido em estímulos físicos e mentais (KILEY-WORTHINGTON, 1977; SAMBAUS, 1985; DANTZER, 1986).

Outros autores concordam com este conceito sobre estereotipia, relatando que são comportamentos anômalos muito observados em animais cativos, mas que não são exibidos por animais em vida livre. Este comportamento não possui um objetivo óbvio e que seguem sempre os mesmos padrões (XENOFONTE, 2006; SCHEPOP, 2008).

Em ruminantes, a estereotipia vista com mais frequência é o lingueteio (tongue-playing) ou hábito de brincar com a língua, jogando-a para fora da boca ou enrolando-a na cavidade oral (REDBO; 1990; REDBO; NORDBLAD 1997; MAREKOVÁ et. al., 2008). 
OLIVEIRA, A.P.G. et al. Uso de enriquecimentos ambientais como mitigadores de comportamentos anormais: uma revisão. PUBVET, Londrina, V. 8, N. 7, Ed. 256, Art. 1697, Abril, 2014.

Paranhos da Costa (1998) classifica os comportamentos anormais como sendo os demonstrados na Tabela 3.

Tabela 3. Definição dos comportamentos anormais.

\begin{tabular}{|c|c|}
\hline $\begin{array}{l}\text { COMPORTAMENTO } \\
\text { ANORMAL }\end{array}$ & DEFINIÇÃO \\
\hline Estereotipias & Balançar o corpo para frente e enrolar a língua; \\
\hline Comportamentos autodestrutivos & $\begin{array}{l}\text { Automutilação, lamber e comer o seu próprio pelo ou } \\
\text { lã; }\end{array}$ \\
\hline Apetite depravado & Ingestão de madeira, cama, terra, fezes; \\
\hline Polidipsia & Ingestão excessiva de água; \\
\hline Agressividade exagerada & Cabeçada e empurrões sem causa definida; \\
\hline Falhas comportamentais & $\begin{array}{l}\text { Cio silencioso, impotência sexual e rejeição de } \\
\text { filhotes; }\end{array}$ \\
\hline Reatividade anormal & $\begin{array}{l}\text { Apatia, inatividade, hiperatividade, reações de } \\
\text { pânico. }\end{array}$ \\
\hline
\end{tabular}

Fonte: adaptada de Paranhos da Costa (1998).

\subsection{Causas de estereotipias}

Os animais domesticados são submetidos à troca de um ambiente natural com condições de estresse reduzido para um meio restrito, sem a possibilidade de pastagem e elevada densidade populacional, que afeta a movimentação dos indivíduos, além da imposição de elementos criados pelo homem, como ordenhadeira, currais, trator, lama e agulhadas, que por serem fatores estressantes acarretam em comportamentos incomuns principalmente quando associados a erros na alimentação (MALAFAIA et. al., 2011).

Todas essas situações podem causar frustrações que norteiam as estereotipias nos seres vivos (DANTZER, 1986) e vários estudos demonstraram que essas ocorrem quando há uma redução da variedade de repertórios comportamentais e um decréscimo do número de ações diferenciadas em um dado comportamento (STOLBA et. al., 1983). 
OLIVEIRA, A.P.G. et al. Uso de enriquecimentos ambientais como mitigadores de comportamentos anormais: uma revisão. PUBVET, Londrina, V. 8, N. 7, Ed. 256, Art. 1697, Abril, 2014.

\section{Enriquecimento ambiental}

Uma das áreas de estudos e emprego dos princípios do comportamento animal é o uso de enriquecimento ambiental, que embora seja resultado de uma preocupação antiga, é de recente utilização (SHEPHERDSON et. al., 2001).

O tema enriquecimento ambiental teve sua significância reconhecida em primeira instância em 1925 por Yerkes e discutida posteriormente em 1950 por Hediger (PIZZUTTO; SGAI; GUIMARÃES, 2009).

Segundo Yerkes (1925) o animal precisa trabalhar para sobreviver, caso isso não ocorra, ele deve ter ao menos a oportunidade de exercitar reações diversas frente a objetos colocados em seu ambiente.

De acordo com Hediger (1950) essas atividades são treinos que servem como terapia ocupacional, pois desperta nos cativos a possibilidade de vencer desafios físicos e mentais.

Porém, determinar um ambiente ideal, com todas essas características é uma tarefa complicada, pois para tanto, deve-se levar em consideração as necessidades comportamentais do animal e estas ainda não são bem definidas (STAFFORD, 2006; YEATES, 2011).

$\mathrm{Na}$ tentativa de se adequar ao ambiente, os animais começam a disputar pelos elementos presentes nesse recinto, e em função disso, passam a desenvolver comportamentos de disputa. Por esta razão, o ambiente deve ser alterado de forma que, segundo Starfford (2006), beneficie ambos os envolvidos.

Neste contexto, pode-se inferir que enriquecimentos ambientais são modificações no ambiente físico ou social do animal combinando conhecimento prévio do habitat e do comportamento natural ou típico desse individuo, visando sua melhoria ou prevalência, bem como a redução dos níveis de estresse, melhoria na saúde e desempenho reprodutivo (BOERE, 2001).

Os enriquecimentos ambientais podem ser ofertados das mais diversas formas, tais como a inserção de objetos (pedaços de madeira, cordas, folhas, 
OLIVEIRA, A.P.G. et al. Uso de enriquecimentos ambientais como mitigadores de comportamentos anormais: uma revisão. PUBVET, Londrina, V. 8, N. 7, Ed. 256, Art. 1697, Abril, 2014.

escadas, rochas, brinquedos e etc), alimentação ofertada de forma diferenciada (escondida, pendurada, congelada, cortada ou inteira, itens novos, horários diferentes) e o estímulo dos sentidos através de essências, brinquedos, gustação e outros (ALMEIDA; MARGARIDO; MONTEIRO FILHO, 2008; PINHEIRO, 2009; CAMPOS et. al., 2010; MENDES et. al., 2011).

Muitos Zoológicos têm utilizado essa técnica, objetivando tornar o recinto mais interativo, uma vez que o aparato torna-se uma novidade e um desafio que simula situações que ocorreriam na natureza, podendo, dessa forma, minimizar os efeitos do cativeiro sobre esses indivíduos (BOERE, 2001).

Bloomsmith et. al. (1991) descreveu os cinco principais tipos de enriquecimentos ambientais e suas subdivisões, conforme apresentado na Tabela 4.

\section{Influência do enriquecimento ambiental no comportamento anormal e bem-estar de animais cativos}

Os enriquecimentos ambientais são utilizados para o aumento do bemestar e o indicativo da eficácia dessa técnica é a redução de comportamentos anormais (WILSON, 1982) e o desenvolvimento de atividades típicas da espécie (NOVAK; SUOMI, 1988).

Mudanças de comportamentos são resultados positivos alcançados após a inserção de enriquecimentos ambientais (MAKI et. al., 1989), pois esta prática torna o ambiente mais estimulante, auxiliando no processo contrário a apatia de cativeiro (ALMEIDA, 2006).

O enriquecimento ambiental é um princípio de manejo, que aumenta a qualidade de vida do animal em cativeiro, levando-o a alcançar um elevado grau de bem-estar psíquico e fisiológico, reduzindo o estresse e estimulando comportamentos típicos da espécie (SHEPHERDSON et. al., 1998; CARLSTEAD; SHEPHERDSON, 2000; BOERE, 2001; HOHENDORFF, 2003; NORDI et. al., 2006). 
OLIVEIRA, A.P.G. et al. Uso de enriquecimentos ambientais como mitigadores de comportamentos anormais: uma revisão. PUBVET, Londrina, V. 8, N. 7, Ed. 256, Art. 1697, Abril, 2014.

Tabela 4. Tipos de enriquecimento ambiental.

\begin{tabular}{|c|c|}
\hline $\begin{array}{l}\text { ENRIQUECIMENTO } \\
\text { AMBIENTAL } \\
\end{array}$ & SUBDIVISÕES \\
\hline Social & $\begin{array}{l}\text { Com contato } \\
\text { - Coespecífico: pares, grupos, temporariamente, } \\
\text { - Intermanentemente; } \\
\text { - Sem contatífo: humanos e não humanos. } \\
\text { - Visão, audição, dispositivo cooperativo; } \\
\text { - Humano e não humano. }\end{array}$ \\
\hline Cognitivo & $\begin{array}{l}\text { - Psicológico: quebra-cabeça, controle do } \\
\text { enriquecimento; } \\
\text { - Exercícios: dispositivos mecânicos, corrida. }\end{array}$ \\
\hline Físico & $\begin{array}{l}\text { - Tamanho; } \\
\text { - Complexidade. } \\
\text { - Internos; } \\
\text { - Permanente (mobília); } \\
\text { - Temporiório (brinquedos, cordas); } \\
\text { - Externos (quebra-cabeças). }\end{array}$ \\
\hline Sensorial & $\begin{array}{l}\text { - Visual (fitas, televisão, imagens, janelas); } \\
\text { - Audição (música, visualizações); } \\
\text { - Outros estímulos (olfato, tato, gosto). }\end{array}$ \\
\hline Nutricional & $\begin{array}{l}\text { - Entrega (frequência, horário, apresentação, } \\
\text { processamento); } \\
\text { - Tipo (novidade, variedade, ramalhetes). }\end{array}$ \\
\hline
\end{tabular}

Fonte: adaptada de Bloomsmith et. al. (1991).

A diminuição de comportamentos anormais e o desenvolvimento de desempenhos naturais da espécie são indicadores da eficiência das técnicas de enriquecimento ambiental (NEWBERRY, 1995). 
OLIVEIRA, A.P.G. et al. Uso de enriquecimentos ambientais como mitigadores de comportamentos anormais: uma revisão. PUBVET, Londrina, V. 8, N. 7, Ed. 256, Art. 1697, Abril, 2014.

Por tudo isso, a utilização de enriquecimentos ambientais é importante, pois torna o recinto mais interessante e educacional para o público, ajuda na conservação da espécie, incentiva comportamentos naturais e aumenta as taxas reprodutivas, além de melhorar o bem-estar físico e psicológico dos animais (CELOTTI, 2001).

Por fim, recomenda-se a inserção de enriquecimentos em cativeiros como técnica auxiliadora na diminuição da ocorrência de comportamentos estereotipados (SANS, 2008).

\section{Conclusão}

Ambientes empobrecidos em estímulos físicos e mentais levam a uma alteração negativa no quadro de estresse de animais cativos, estimulando o desenvolvimento de comportamentos anormais ou atípicos da espécie. Com a implementação de enriquecimentos ambientais ocorre a redução do estresse e, consequentemente, a elevação do grau de bem-estar do indivíduo, que passa a concentrar seus padrões comportamentais naqueles normais ou naturais da espécie.

\section{Referências Bibliográficas}

ALMEIDA, A. M. R. Influência do enriquecimento ambiental no comportamento de primatas do gênero Ateles em diferentes condições de cativeiro no Departamento Zoológico de Curitiba, PR. Monografia para obtenção do grau de bacharel em Ciências Biológicas pela Universidade Federal do Paraná, Curitiba, 2006.

ALMEIDA, A. M. R.; MARGARIDO, T. C. C.; FILHO, E. L. A. Influência do enriquecimento ambiental no comportamento de primatas do gênero Ateles em cativeiro. Arquivos de Ciências e Zologia, Umuarama, v. 11, n. 2, p. 97-102, 2008.

BLOOMSMITH, M. A.; BRENT, L. Y.; SCHAPIRO, S. J. Guidelines for developing and managing an environmental enrichment program for nonhuman-primates. Laboratory Animal Science, v. 41, p.327-7, 1991.

BOERE, V. Behavior and environment enrichment. IN: Fowler ME, Cubas ZS. Biology, medicine and surgery of South American wild animals. Ames, IA: Iowa University Press, 2001. p.263-266.

BOSSO, P. L. Comportamento Anormal. Disponível em: <http://www.zoologico.sp.gov.br/peca9.htm>.Acesso em: 13 abril. 2013. 
BROOM, D. Animal welfare: concepts and measurements. Journal of Animal Science, v. 69, p. 4167-4175, 1991.

BROOM, D.M. Coping with challenge: welfare in animals including humans. Berlin: Dahlem University Press, 2001.

BROOM, D.M.; FRASER, A.F. Comportamento e bem-estar de animais domésticos. 4. ed. Barueri/SP: Manole, 2010.

BROOM, D. M.; JOHNSON, K. G. Stress and Animal Welfare. Reimpressão. Netherlands: Kluwer, 2000. 211 p. 9.

BROOM, D. M.; MOLENTO, C.F.M. BEM-ESTAR ANIMAL: CONCEITO E QUESTÕES RELACIONADAS - REVISÃO. Archives of Veterinary Science, v. 9, n. 2, p. 1-11, 2004.

BURN, C. C.; DENNISON, T. L.; WHAY, H. R. Relationships between behaviour and health in working horses, donkeys, and mules in developing countries. Applied Animal Behaviour Science, v. 126, p. 109-118, 2010.

BUTTERWORTH, A.; MENCH, J. A.; WIELEBNOWSKI, N. Practical strategies to assess (and improve) welfare. In: APPLEBY, M. C.; MENCH, J. A.; OLSSON, I. A. S. Animal Welfare. 2 ed. Nosworthy Way: Wallingford, UK, p. 200-214, 2011.

CAMPOS, J. A.; TINÔCO, I. de F. F.; SILVA, F. F.; PUPA, J. M. R.; SILVA, I. J. O. Enriquecimento ambiental para leitões na fase de creche advindos de desmame aos 21 e 28 dia. Revista Brasileira de Ciências Agrárias, v.5, n.2, p. 272-278, 2010.

CARLSTEAD, K.; SHEPHERDSON, D. Alleviating stress in zoo animals with environmental enrichment. In: MOBERG, G.P.; MENCH, J.A. The Biology of animal stress: basic principles and implications for animal welfare. [S.1.]: CAB International, Cap. 16, p. 337$354,2000$.

CELOTTI, S. Guia para o enriquecimento das condições ambientais de cativeiro. Universities Federation for Animal Welfare. England. 2001. p. 337.

COCKRAM, M. S.; HUGHES, B. O. Health and Disease. In: APPLEBY, M. C.; MENCH, J. A.; OLSSON, I. A. S. Animal Welfare. 2 ed. Nosworthy Way: Wallingford, UK, p. 120-137, 2011.

DANTZER, R. Behavioral physiological and functional aspects of stereotyped behavior: A review and a re-interpretation. Journal of Animal Science, v.62, p.1776-1786, 1986.

FRASER, A. F.; BROOM, D.M. Farm animal behaviour and welfare. Oxon: CABI, p. 437, 2002.

HAMMERSCHMIDT, J. Desenvolvimento e aplicação de perícia em bem-estar animal. Curitiba, 2012. Dissertação (Mestrado em Ciências Agrárias) - Universidade Federal do Paraná, Paraná, 2010.

HEDIGER H. Wild animals in captivity. London: Butterworths, 1950, 207p.

HOHENDORFF, R.V. Aplicação e avaliação de enriquecimento ambiental na manutenção de bugio ( Alouatta spp LACÉPEDE, 1799) no Parque Zoológico de Sapucaia do SulRS. Dissertação de Mestrado - Universidade Federal do Rio Grande do Sul, Porto Alegre, 2003.

KILEY-WORTHINGTON, M. Behavioural problems of farm animals. Oriel Press, Stocksfield, Engl. p.211, 1977. 
KYRIAZAKIS, I; TOLKAMP, B. Hunger and Thirst. In: APPLEBY, M. C.; MENCH, J. A.; OLSSON, I. A. S. Animal Welfare. 2 ed. Nosworthy Way: Wallingford, UK, p. 44-63, 328 p. 2011. MACHADO FILHO, L.C.P.; HOTZEL, M.J. Bem-estar dos suínos. In: Seminário Internacional de Suinocultura, 5., 2000, São Paulo-SP. Anais... Concórdia: Embrapa Suínos e Aves, 2000. p. 70-83.

MADELLA-OLIVEIRA, A. F.; QUIRINO, R. C.; RUIZ-MIRANDA, C. R.; FONSECA, F. A. O processo de domesticação no comportamento dos animais de produção. PUBVET, Londrina, v. 5, n. 31, Ed. 178, Art. 1204, 2011.

MAESTRIPIERI, D.; SCHINO, G.; AURELI, F.; TROISI, A. A modest proposal: displacement activities as an indicator of emotions in primates. Animal Behavior, v. 44, p. 967-979, 1992.

MAKI S, ALFORD PL, BLOOMSMITH MA, FRANKLIN J. Food puzzle device simulation termite fishing for captive chimpanzee (Pan troglodytes). Am J Primatol, v.1, p.71-78, 1989.

MALAFAIA, P.; BARBOSA, J. D.; TOKARNIA, C. H. Distúrbios comportamentais em ruminantes não associados a doenças: origem, significado e importância. Pesquisa Veterinária Brasileira, Rio de Janeiro, v. 31, n. 9, p. 781-790, 2011.

MARANHÃO, R. P. A.; PALHARES, M. S.; MELO, U. P.; REZENDE, H. H. C.; BRAGA, C. E.; SilVA FILHO, J. M.; VASCONCELOS, M. N. F. Afeç̧ões mais freqüentes do aparelho locomotor dos eqüídeos de tração no município de Belo Horizonte. Arquivo Brasileiro de Medicina Veterinária e Zootecnia, v. 58, p. 21-27, 2006.

MAREKOVÁ, J.; KOTTFEROVÁ, J.; JAKUBA T.; ONDRASOVICOVA, O.; ONDRASOVIC, M. Orosthenic syndrome in adult cattle: A minireview. Folha Veterinaria. v. 52, p.75-76, 2008.

MASON, G. J. Stereotypies: a critical review. Animal Behavior, v. 41, p.1015-1037, 1991.

$\mathrm{MENCH}$, J. A. Farm animal welfare. In. BEKOFF, M. Encyclopedia of Animal Rigths and Animal Welfare. Connecticut: Greenwood, p.170-171, 1998.

MENDES, R.C.; FARIA, H.A.de; SALVIO, G.M.M. Enriquecimento ambiental para leitões na fase de creche no IF Sudeste MG-campus Barbacena. SIMPÓSIO DE PESQUISA E INOVAÇÃO, 2.; SEMINÁRIO DE INICIAÇÃO CIENTÍFICA,1., Anais...Barbacena: IF Sudeste MG, p.22-24, 2011.

MENDL, M.; BURMAN, O.; LAUGHLIN, K. e PAUL, E. Animal memory and animal welfare. Animal welfare, v.10, p.141-159, 2001.

MENDONÇA-FURTADO, O. Uso de ferramentas como Enriquecimento ambiental para macacos-prego (Cebus apella) cativos. São Paulo, 2006. Dissertação (Mestrado em Psicologia) - Universidade de São Paulo, 2006.

MOLENTO, C. F. M. BEM-ESTAR E PRODUÇÃO ANIMAL: ASPECTOS ECONÔMICOS - REVISÃO. Archives of Veterinary Science, v. 10, n. 1, p. 1-11, 2005.

MOURA D. J.; BUENO, L. G. F.; LIMA, K. A. O.; CARVALHO, T. M. R.; MAIA, A. P. A. M. Strategies and facilities in order to improve animal welfare. Revista Brasileira de Zootecnia, v.39, p.311-316, 2010.

MUNRO, R.; MUNRO, H. M. C. Animal abuse and unlawful killing: forensic veterinary pathology. China: Saunders, 2008. 106 p. 
NEWBERRY R.C.. Environmental enrichment - increasing the biological relevance of captive environments. Appl. Anim. Behav. Sci. V.44, p.229-243, 1995.

NORDI, W. N.; YAMASHIRO, K.; KLANK, M.; CARDOZO, E. C.; DAHLKE, F.; DITRICH, R. L.; MOLENTO, C. F. M. Impacto da oferta de poleiros sobre o bem-estar de frangos de corte. Archives of Veterinary Science, v. 11, n. 3, p. 19-25, 2006.

NOVAK M. A.; SUOMI, S. Psychological well-being of primates in captivity. Am Psychol, v.43, p.765-773, 1988.

OIE. Código Sanitario para Los Animales Terrestres. 21. ed. OIE, 2012. Disponível em: http://www.oie.int/index.php?id=169\&L=2\&htmfile=chapitre_1.7.1.htm. Acesso em: $12 / 04 / 2013$.

PARANHOS DA COSTA, M. J. R., ANDRIOLO, A. Amamentação e Alo-amamentação em Búfalos (Bubalus bubalis). In: M. J. R. PARANHOS DA COSTA; CROMBERG V. U. (eds). Comportamento Materno em mamíferos (Bases Teóricas e Aplicações aos Ruminantes Domésticos), Ribeirão Preto - SP, p. 247-261, 1998.

PINHEIRO, J. V. A pesquisa com bem estar animal tendo como alicerce o enriquecimento ambiental através da utilização de objeto suspenso no comportamento de leitões desmamados e seu efeito como novidade. Dissertação (Mestrado) - Faculdade de Medicina Veterinária e Zootecnia, Universidade de São Paulo, Pirassununga, 2009.

PINHEIRO MACHADO, L. C.; BRIDI A. M.; HÖTZEL M. J. Ética na produção animal. Zootec 2007 - A zootecnia frente a novos desafios. Livro de resumos, p. 3-16.

PIZZUTO, C. S.; SGAI, M. G. F. G.; GUIMARÃES, M. A. B. V. O enriquecimento ambiental como ferramenta para melhorar a reprodução e o bem-estar de animais cativos. Revista Brasileira de Reprodução Animal, Belo Horizonte, v.33, n. 3, p.129-138, 2009.

RAMOS, J. B. Bem-estar animal - A ciência de respeito aos animais. Informativo do Instituto Ecológico Aqualung. No 68, ano XII, Julho/agosto, 2006.

REDBO, I. Changes in duration and frequency of stereotypies and their adjoining behaviours in heifers, before, during and after the grazing period. Applied. Animal. Behaviour. Science. v. 26, p.57-67, 1990.

REDBO, I.; NORBLAND, A. Stereotypies in heifers are affected by feeding regime. Applied Animal Behaviour Science. v.53, p.193-202, 1997.

SAMBRAUS H. H. Mouth-based anomalous syndromes. In:Fraser A. F. (Ed.), Ethology of Farm Animals. Elsevier, Amsterdam, p.991-422, 1985.

SANS, E. C. O. Enriquecimento ambiental no zoológico municipal de curitiba/PR. Curitiba, 2008.

SGAI, M. G. F. G. Avaliação da influência das técnicas de enriquecimento ambiental nos parâmetros endócrinos e comportamentais de Callithrix penicillata (sagui-detufospretos) mantidos em estabilidade social e isolados. 2007. p.113. Dissertação (Mestrado em Reprodução Animal) - Universidade de São Paulo, São Paulo, 2007.

SHEPHERDSON, D.J. Tracing the path of environmental enrichment in zoos. In SHEPHERDSON, D.J.; MELLEN, J.D.; HUTCHINS, M. (Eds.). Second Nature: environmental enrichment for captive animals. Washington D.C.: Smithsonian Institution Press, cap. 1, 1998. p.1-12. 
SHEPHERDSON, D. J.; MELLEN, J. D.; HUTCHINS, M. Second Nature: environmental Universities Federation for Animal Welfare. England. 2001. p. 337.

SILVA, I.J.O.; PANDORFI, H.; PIEDADE, S.M.S. Influência do sistema de alojamento no comportamento e bem- estar de matrizes suínas em gestação. Revista Brasileira de Zootecnia. V.37, n.7, p.1319-1329, 2008.

STAFFORD, K. J. The welfare of dogs. Springer, Dordrecht, The Netherlands. p. 8-11. 2006.

STOLBA, A.; BAKER, N.; WOOD-GUSH, D. G. M. 1983. The characterization of stereotyped behaviour in stalled sows by informational redundancy. Behaviour, Leiden, v. 87, n. 3-4, p. 157-182, 1983.

WILSON S. F. Environmental influences on the activity of captive apes. In: Zoo Biology, v.1, p.201-209, 1982.

XENOFONTE, P. F.; OLIVEIRA, R. L.; CRUZ, M. A. O. M. Análise Comportamental de um casal de Panthera tigris tigris (Linnaeus, 1758) do Parque Dois Irmãos. In: Encontro De Bioética E Bem Estar Animal Do Agreste Meridional Pernambucano,1., 2006, Garanhuns. Pôsteres. Recife: UFRPE/UAG, 2006.

YERKES, R. M. Almost human. London: Jonathan Cope, 1925. p.229.

YEATES, J. W. Maximising canine welfare in veterinary practice and research: a review. The Veterinary Journal, 2011. 